LAWRENCE LIVERMORE NAT IO N A L LABORATORY

\title{
Fermion Monte Carlo calculations on liquid-3He
}

M. H. Kalos, L. Colletti, F. Pederiva

June 8, 2004

Hypernodal Quantum Monte Carlo Trento, Italy July 5, 2004 through July 9, 2004 
This document was prepared as an account of work sponsored by an agency of the United States Government. Neither the United States Government nor the University of California nor any of their employees, makes any warranty, express or implied, or assumes any legal liability or responsibility for the accuracy, completeness, or usefulness of any information, apparatus, product, or process disclosed, or represents that its use would not infringe privately owned rights. Reference herein to any specific commercial product, process, or service by trade name, trademark, manufacturer, or otherwise, does not necessarily constitute or imply its endorsement, recommendation, or favoring by the United States Government or the University of California. The views and opinions of authors expressed herein do not necessarily state or reflect those of the United States Government or the University of California, and shall not be used for advertising or product endorsement purposes. 


\title{
Fermion Monte Carlo calculations on liquid- ${ }^{3} \mathrm{He}$
}

\author{
M. H. Kalos ${ }^{1}$, L. Colletti ${ }^{2,3}$, and F. Pederiva ${ }^{2,4}$ \\ ${ }^{1}$ Lawrence Livermore National Laboratory, \\ University of California, Livermore, California 94551 \\ ${ }^{2}$ Dipartimento di Fisica, Università di Trento, 38050 Povo(TN), Italy \\ ${ }^{3}$ Dep. of Computer Science, Free University of Bolzano, 39100 Bolzano, Italy \\ ${ }^{4}$ DEMOCRITOS - INFM National Simulation Center, Trieste, Italy
}

Methods and results for calculations of the ground state energy of the bulk system of ${ }^{3} \mathrm{He}$ atoms are discussed. Results are encouraging: we believe that they demonstrate that our methods offer a solution of the "fermion sign problem" and the possibility of direct computation of many-fermion systems with no uncontrolled approximations. Although the method is still rather inefficient compared to variational or fixed-node methods, we have been able to obtain useful answers.

PACS numbers: 67.55.-s, 02.70.Ss

\section{INTRODUCTION}

Powerful Monte Carlo methods ${ }^{1}$ can provide estimates of ground state properties of bosonic many-body systems that are not subject to uncontrolled approximations. But the mapping of the many-body quantum wavefunction to a probability distribution function faces severe challenges when considering the global antisymmetry that a fermionic wavefunction must satisfy. The methods that we have adopted to face this "fermion sign problem" 2 are based on a population of pairs of positive and negative walkers which is generated using Diffusion Monte Carlo modified in the following ways:

(1) Different guiding functions are used for walkers of different signs;

(2) When walkers of different signs branch differently (as they must) new pairs are created;

(3) The "diffusion" steps of the walkers in a pair are correlated;

(4) Walkers in a pair that come close can be canceled in a way that guarantees that the future value of any projection with an antisymmetric 
function is exactly preserved;

(5) We have been able to adapt the technique of "acceptance-rejection" to our method. We have also experimented with a "Second-stage" importance sampling scheme, in which a function of the coordinates of both walkers of a pair is used to modify the distribution of the walkers.

\section{THE METHOD}

Let us consider a system of $N$ atoms of which the coordinates of the $i$ th particle are represented by a tridimensional vector $\mathbf{r}_{\mathbf{i}}$. The atoms interact with one another via two- and three-body forces summarized by a potential $V\left(\mathbf{r}_{\mathbf{1}}, \ldots \mathbf{r}_{\mathbf{N}}\right)$. Our primary interest is in the ground state energy $E_{0}$ of the system, for which we need to solve the many-body Schrödinger equation. Diffusion Monte Carlo follows the evolution in imaginary time; using atomic units, this equation translates into

$$
\left[-\frac{1}{2} \nabla^{2}+V(\mathbf{R})-E_{T}\right] \psi(\mathbf{R}, \tau)=-\frac{\partial \psi(\mathbf{R}, \tau)}{\partial \tau}
$$

which is a diffusion and absorption equation, solved by iterating an appropriate Green's function $G\left(\mathbf{R}, \mathbf{R}^{\prime}, \delta \tau\right)$ on the $3 N$-dimensional vector $\mathbf{R}^{\prime}\left(x_{1}, \ldots, x_{3 N}\right)$ that represents a whole configuration ("walker") of the system,

$$
\psi(\mathbf{R}, \tau+\delta \tau)=\int G\left(\mathbf{R}, \mathbf{R}^{\prime}, \delta \tau\right) \psi\left(\mathbf{R}^{\prime}, \tau\right) d \mathbf{R}^{\prime}
$$

At each step $\psi(\mathbf{R}, \tau)$ is represented by an ensemble of discrete points $\left\{\mathbf{R}_{m}\right\}$ that are then advanced and reproduced or removed according to $G\left(\mathbf{R}, \mathbf{R}^{\prime}, \delta \tau\right)$. In the limit of sufficiently long imaginary-time $\tau$, only the term with the lowest energy survives

$$
\psi(\mathbf{R}, \tau)=N_{0} e^{-\tau\left(E_{0}-E_{T}\right)} \phi_{0}(\mathbf{R})
$$

and, if $E_{T}=E_{0}$, the walkers $\left\{\mathbf{R}_{m}\right\}$ are sampled from a stationary probability density distribution that maps the symmetric ground state $\phi_{0}(\mathbf{R})$.

In order to improve the efficiency of this filtering of the solution, one introduces a known trial wavefunction $\psi_{T}(\mathbf{R})$ with the aim of changing the sampling process in a known way ${ }^{3}$ while preserving averages. In our FMC algorithm this concept is split into two separate auxiliary functions: a strictly positive guiding function $\psi_{G}(\mathbf{R})$ whose role is to modify the random walk, while an antisymmetric trial function $\psi_{T}(\mathbf{R})$ that should resemble the solution as closely as possible is used to project out quantities of interest, such 
as the local energy $E_{T}$. Thus the density of walkers generated by the walk will be

$$
f(\mathbf{R}, \tau)=\psi_{G}(\mathbf{R}) \psi(\mathbf{R}, \tau) \underset{\tau \rightarrow \infty}{\longrightarrow} \psi_{G}(\mathbf{R}) \phi_{0}(\mathbf{R})
$$

We separate now the set $\left\{\mathbf{R}_{\mathbf{m}}\right\}$ of walkers into two subsets of walkers $\left\{\mathbf{R}_{m}^{+}\right\}$, $\left\{\mathbf{R}_{m}^{-}\right\}$that respectively add or subtract their contributions to statistical expectations such as the energy

$$
E_{0}=\frac{\sum_{m}^{N_{p}}\left[\frac{\hat{H} \psi_{T}\left(\mathbf{R}_{m}^{+}\right)}{\psi_{G}\left(\mathbf{R}_{m}^{+}\right)}-\frac{\hat{H} \psi_{T}\left(\mathbf{R}_{m}^{-}\right)}{\psi_{G}\left(\mathbf{R}_{m}^{-}\right)}\right]}{\sum_{m}^{N_{p}}\left[\frac{\psi_{T}\left(\mathbf{R}_{m}^{+}\right)}{\psi_{G}\left(\mathbf{R}_{m}^{+}\right)}-\frac{\psi_{T}\left(\mathbf{R}_{m}^{-}\right)}{\psi_{G}\left(\mathbf{R}_{m}^{-}\right)}\right]}
$$

where $N_{p}$ is the number of pairs of configurations that enter the averaging. If the signed walkers independently sample the configuration space according to the distribution (4) they will lead to the fermion decay. In particular the denominator of Eq. (5) will be unstable since the $\left\{\mathbf{R}_{m}^{+}\right\}$and the $\left\{\mathbf{R}_{m}^{-}\right\}$ will be symmetrically distributed, averaging their contributions around zero. The incorporation of a correlation between pairs of walkers in the randomwalk process has been shown to be effective in avoiding this decay of the signal-to-noise ratio that regularly occurs in a fermionic DMC ${ }^{4,5}$. Before introducing correlation, we first start by distinguishing the signed walkers by means of different $\psi_{G}$ 's which we call $\psi_{G}^{ \pm}$respectively.

In FMC we introduce two functions $\psi_{G}^{ \pm}$which bias differently the populations of plus and minus walkers and thus act as symmetry breaking fields. The ingredients of our guiding functions are Slater Determinants $D^{\uparrow(\downarrow)}$ of like-spin single particle wavefunctions, and two symmetric Jastrow products, $J^{S}(\mathbf{R})$ and $J^{A}(\mathbf{R})$ with different parameters. We now construct a function antisymmetric under permutation of atom labels

$$
\psi_{A}(\mathbf{R})=J^{A}(\mathbf{R}) D^{\uparrow}(\mathbf{R}) D^{\downarrow}(\mathbf{R})
$$

and a symmetric one

$$
\psi_{S}(\mathbf{R})=J^{S}(\mathbf{R})
$$

Finally, the guiding functions themselves are constructed by combining (6) and (7):

$$
\psi_{G}^{ \pm}(\mathbf{R})=\sqrt{\psi_{S}^{2}(\mathbf{R})+c^{2} \psi_{A}^{2}(\mathbf{R})} \pm c \psi_{A}(\mathbf{R})
$$

with $c$ a small and adjustable parameter. The functions $\psi_{G}^{ \pm}$are positive for any $\mathbf{R}$, as is necessary for an importance function. For an odd permutation of particles in a configuration one has that the biased distributions of the two signed walkers map into each other. 
The orbitals of our Slater determinants comprise the standard backflow function $^{6}$, while the Jastrow products provide two-body correlations via a pseudopotential of the McMillan type and three-body correlations.

The diffusion process for the distribution (4), is generalized as follows: the two $\psi_{G}^{ \pm}$are applied separately to drift the walkers of a pair from their old positions $\mathbf{R}_{o}^{+}, \mathbf{R}_{o}^{-}$:

$$
\mathbf{R}_{d}^{ \pm}=\mathbf{R}_{o}^{ \pm}+2 \delta \tau \frac{\nabla \psi_{\mathbf{G}}^{ \pm}\left(\mathbf{R}_{\mathbf{o}}^{ \pm}\right)}{\psi_{G}^{ \pm}\left(\mathbf{R}_{o}^{ \pm}\right)} .
$$

We make use of correlated dynamics in the pairs diffusion. The branching of the pairs is carried out taking into account the possibility of an overlap of the members of a pair, which leads to a partial cancellation of the contribution of the pair to the future statistics. Since the weights $\omega\left(\mathbf{R}^{ \pm}\right)$of the single walkers in a pair can be different at each generation, a mechanism for repairing the lone walker must be introduced. Details of this algorithm can be found in Refs. ${ }^{7,8}$

Quantities of interest, such as the total energy, are projected out by using a trial wave function. In our FMC method that role is played by the antisymmetric function $\psi_{A}$ of Eq. (6). To account for the branching process, each pair may contribute once weighted with $\omega\left(\mathbf{R}_{m}^{ \pm}\right)$and Eq. (5) then becomes:

$$
E_{0}=\frac{\sum_{m}^{N_{p}}\left[\frac{\hat{H} \psi_{A}\left(\mathbf{R}_{m}^{+}\right)}{\psi_{G}\left(\mathbf{R}_{m}^{+}\right)} \omega\left(\mathbf{R}_{m}^{+}\right)-\frac{\hat{H} \psi_{A}\left(\mathbf{R}_{m}^{-}\right)}{\psi_{G}\left(\mathbf{R}_{m}^{-}\right)} \omega\left(\mathbf{R}_{m}^{-}\right)\right]}{\sum_{m}^{N_{p}}\left[\frac{\psi_{A}\left(\mathbf{R}_{m}^{+}\right)}{\psi_{G}\left(\mathbf{R}_{m}^{+}\right)} \omega\left(\mathbf{R}_{m}^{+}\right)-\frac{\psi_{A}\left(\mathbf{R}_{m}^{-}\right)}{\psi_{G}\left(\mathbf{R}_{m}^{-}\right)} \omega\left(\mathbf{R}_{m}^{-}\right)\right]} .
$$

In order to improve the efficiency of our method we introduced and experimented with several classes of technical improvements.

The first was an adaptation to the correlated diffusion of pairs of walkers of the "Acceptance/Rejection" method widely used in fixed-node computations ${ }^{9,10}$.

The second was a class of refinements that we referred to collectively as "second-stage importance functions." Here we took note of the general character of importance sampling for random walks, namely that the stochastic dynamics should be biased by a function proportional to the chance that a walker contributes to the asymptotic distribution, namely the steady state of the population. That steady state can be measured by the denominator of the energy quotient. Quite clearly, the needed function depends on the positions of both of our walkers, a fact not accounted for in the method described above.

We tried several forms of this second-stage importance functions with and without Acceptance/Rejection, and found significant but not decisive 

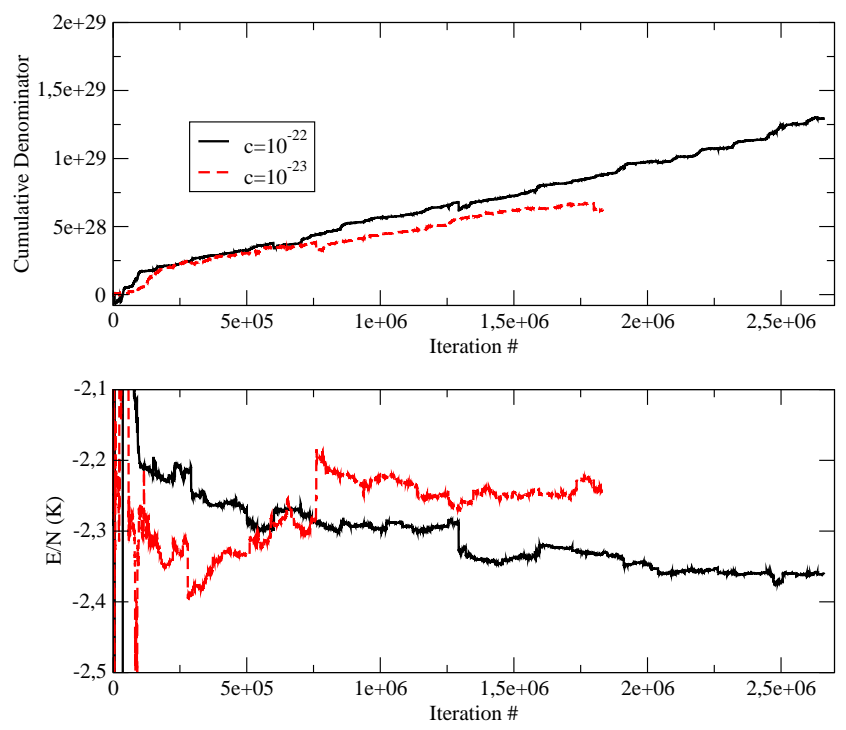

Fig. 1. Effect of the amount of antisymmetry (measured by $c$ in Eq. (8)) in the guiding function. Top: cumulative denominator; Bottom: cumulative energy. These runs have been obtained with Acceptance/Rejection, denominator-weighted SSIS and $\delta \tau=6 \times 10^{-5}$

improvements. Nevertheless, we believe that the path to much better efficiency lies in better importance sampling of the dynamics and are actively pursuing new ideas.

\section{RESULTS}

Table 1 shows average results for the various runs. In particular it is shown that even with a small population of 192 pairs, a long FMC run with SSIS and Acceptance/Rejection procedure gives results that are comparable with the fixed node ones. Figure 1 shows the behavior of a typical run for 54 ${ }^{3} \mathrm{He}$ atoms. Top panel shows the behavior of the cumulative denominator. The growth is an indication of the stability of the run. In the bottom panel the cumulative value of the projected energy is shown. As can be seen, fluctuations are rather large but a clear converging behavior emerges. 
Table 1. FMC results with respect to parameters used. "A/R" refers to acceptance/rejection and "SSIS" to the kind of second-stage importance function used. $\tau_{r e l}$ denotes the relaxation time for the run. Note that no extrapolation to zero time step has been calculated. Bottom line: fixed node result.

\begin{tabular}{ccccccc}
$N_{p}$ & $c$ & $\delta \tau$ in $K^{-1}$ & $A / R$ & $S S I S$ & length in $\tau_{r e l}$ & $E(\Delta E)$ in $K$ \\
\hline 192 & $10^{-23}$ & $6 \times 10^{-5}$ & yes & denom & 16 & $-2.24(3)$ \\
192 & $10^{-23}$ & $6 \times 10^{-5}$ & yes & no & 20 & $-2.26(13)$ \\
192 & $10^{-22}$ & $6 \times 10^{-5}$ & yes & eucl & 11 & $-2.215(12)$ \\
192 & $10^{-22}$ & $6 \times 10^{-5}$ & no & denom & 25 & $-2.24(14)$ \\
192 & $10^{-22}$ & $6 \times 10^{-5}$ & yes & denom & 25 & $-2.38(3)$ \\
\hline 192 & FXN & $6 \times 10^{-5}$ & yes & denom & 10 & $-2.369(2)$
\end{tabular}

\section{ACKNOLEDGMENTS}

We gratefully thank David Ceperley, Geoffrey Chester, Berni Alder, Joseph Carlson, Kevin Schmidt, and Randolph Hood for stimulating comments. We are also indebted to David Hardin for a critical review of the manuscript. This work was performed under the auspices of the U.S. Department of Energy by the University of California, Lawrence Livermore National Laboratory under contract No. W-7405-Eng.-4, and funded by the Laboratory Directed Research and Development (LDRD) Program at Lawrence Livermore National Laboratory (LLNL) under project 01-LW-040.

\section{REFERENCES}

1. D. M. Ceperley, Rev. Mod. Phys. 78, 279 (1995).

2. J. B. Anderson, J. Chem. Phys. 63, 1499 (1975).

3. M. H. Kalos, D. Levesque and L. Verlet, Phys. Rev. A 9 , 2178 (1974).

4. M. H. Kalos, Phys. Rev. E 53, 5420 (1996).

5. Z. Liu, S. Zhang and M. H. Kalos, Phys. Rev. E 50, 3220 (1994).

6. K. E. Schmidt, M. A. Lee, M. H. Kalos and G. V. Chester, Phys. Rev. Lett. 47, 807 (1995).

7. M. H. Kalos and F. Pederiva, Proceedings of the NATO-ASI Conference Quantum Monte Carlo methods in Phy sics and Chemistry, ed. by M. P. Nightingale and C. J. Umrigar, Kluwer, Dordrecht, The Netherlands (1999)

8. M. H. Kalos and F. Pederiva, Phys. Rev. Lett. 85, 3547 (2000)

9. P. J. Reynolds, D. M. Ceperley, B. J. Alder and W. A. Lester, Jr., J. Chem. Phys. 77, 5593 (1982).

10. C. J. Umrigar, M. P. Nightingale and K. J. Runge, J. Chem. Phys. 99, 2865 (1993). 